\title{
Rare case of gallbladder cancer presenting with meta- stasis to bone and brain in an African American male discovered by F18-FDG PET/ CT
}

\author{
Aung Z. Win, Carina M. Aparici \\ Department of Radiology, Nuclear Medicine section, San Francisco Veteran Affairs Medical Center, San Francisco, CA, USA \\ Correspondence: Aung Zaw Win. Address: Division of Nuclear Medicine, Department of Radiology, San Francisco VA \\ Medical Center, 4150 Clement Street, San Francisco, CA 94121, USA. Telephone: 1-415-2214-810 Ext. 3051. Fax: \\ 1-415-7502-142. Email: Aung.Win2@va.gov.
}

Received: May 15, 2012

DOI : $10.5430 / j b g c . v 3 n 2 p 24$
Accepted: J une 25, 2012

Online Published: February 22, 2013

\section{Abstract}

The most common site of metastasis in gallbladder cancer is the liver. To our knowledge, there are no reports in the literature of gallbladder cancer metastasizing to both the brain parenchyma and the bone. There are only 5 prior reports of gallbladder cancer metastasis to the bone in the English Language literature. Likewise, gallbladder neoplasm metastasizing into the brain parenchyma is extremely rare. We present a rare case of gallbladder cancer presenting with metastases to both the brain parenchyma and the bone in a 71 year old African American male. This patient had early bone metastasis evidenced by hypermetabolic lesions on FDG PET/CT imaging, without density abnormalities of the bone tissue. Gastrointestinal (GI) tumors are commonly detected by CT but for metastasis, F18-FDG PET/CT has higher sensitivity and higher positive predictive value than CT. Since at the initial presentation, most patients suffering from gallbladder cancer tend to have metastatic disease, we raise a question as to whether F18-FDG PET/CT scans should be included in the routine work-up protocol.

\section{Key words}

Gallbladder cancer, F18-FDG PET/CT, Skeletal metastasis, Brain metastasis

\section{Introduction}

Gallbladder cancer is very rare and it accounts for only $2 \%-4 \%$ of all malignant GI tumors ${ }^{[1]}$. The peak incidence is in the seventh decade of life ${ }^{[2]}$. It is an aggressive tumor with early dissemination to lymph nodes. The prognosis is poor except when detected incidentally in early stages. It has a five year survival rate of about 5\% with a median survival of about seven months ${ }^{[1]}$. Its incidence in random autopsies is about $0.4 \%$ and in cholecystectomies about $1 \%$ are incidentally found to have gallbladder cancers ${ }^{[1]}$. The incidence is particularly high among Native American populations. The annual incidence in Native American females is about 75 per 100,000 compared to 2.5 per 100,000 for other Americans ${ }^{[1]}$.

Liver is the most common site of gallbladder cancer metastasis and the skeletal system is the least common site ${ }^{[2,3]}$. At the time of the first diagnosis, 25\% of the gallbladder tumors are localized to the gallbladder wall, 35\% have local nodal involvement and $40 \%$ have distant metastasis ${ }^{[4]}$. The clinical manifestations of the carcinoma of the gallbladder are 
nonspecific. They include right upper quadrant pain, nausea and vomiting, jaundice, weight loss, anorexia, ascites, and abdominal mass ${ }^{[5]}$. Differential diagnoses of the gallbladder tumors are acute or chronic cholecystitis, choledocholithiasis, and pancreatic cancer. Ultrasonography is usually the first exam in diagnosing of the disease and CT is often used for staging the disease. However, a CT scan is not ideal for identifying nodal spread and distant metastases ${ }^{[5]}$.

Surgery is the only curative treatment for gallbladder cancer ${ }^{[6]}$. On the other hand, palliative procedures can be done in unresectable cases with jaundice or duodenal obstruction ${ }^{[6]}$. Other treatment options depend on the stage of the disease and they can only prolong the survival of patients. T2 tumors that invade the perimuscular connective tissue without extension beyond the serosa or into the liver, are treated by an extended cholecystectomy ${ }^{[6]}$. For tumors that grow beyond the serosa and invade the liver and other organs (T3 and T4 tumors), tumor excision with extended right hepatectomy can be done as a palliative treatment ${ }^{[7]}$. Chemotherapy is used in some inoperable cases and the literature reports the use of cisplatinum and fluorouracil combination, gemcitabine based combinations, and Etoposide, adriamycin, and cisplatinum (EAP) combination ${ }^{[8,9]}$. Furthermore, stereotactic radiotherapy has been employed for isolated liver metastasis ${ }^{[10]}$.

\section{Case presentation}

The patient is a 71 year old widowed African American man who used to work in the field maintenance after discharge from the Navy. He was brought to the San Francisco Veterans Affairs Medical Center (SFVAMC), Emergency Department for worsening abdominal pain. According to the patient, he was in his usual state of health until a few months ago when he developed right upper quadrant (RUQ) pain, not related to food intake. He also reports 30lb weight loss over a few months. The patient thought this was due to his dentures as he can only take liquids even though he has an appetite. He states that there is no decrease in energy level and he is able to climb 2 flights of stairs without any shortness of breath or chest pain. In addition, he reports that the back pain between his shoulder blades which has been present for a few years, is worsening. He was taking hydrocodone for the back pain. The patient denies any recent trauma, but he said he fell about $8 \mathrm{ft}$ on his back when he was in the service and no imaging was done back then. The patient denied any other problems.

The patient had a past medical history of Type II diabetes, hypertension and hyperlipidemia. He has a prostate operation 8-9 years ago and a left cataract surgery. He never smokes and he has 1 alcoholic drink every other day on average. His family history is positive for hypertension and heart disease but negative for any type of cancer. Physical exam revealed no abnormalities besides the RUQ abdominal pain.

The patient had a prior CT scan on which the gallbladder appeared abnormal with irregular asymmetric enhancing wall thickening along the nondependent anterior wall, intra and extra hepatic biliary ductal dilatation, cholelithiasis and choledocolithiasis and an enhancing soft tissue lesion in the medial segment of the left hepatic lobe extending towards the porta hepatis.

The Emergency physician referred the patient to Nuclear Medicine on July 11, 2011, to evaluate for possible gallbladder cancer metastasis (Images shown in Figures 1-5). The images showed numerous intensely hypermetabolic bony metastases. For instance, lytic FDG avid lesions are seen in the left ischial tuberosity, left posterior acetabulum, right anterior superior pubic ramus, right anterior ilium (with maximum SUV of 23), right sacral ala, left iliac bone and axial skeleton. Multiple metastases are noted in the bilateral ribs as well as in the inferior tip of the left scapula, superior aspect of the left scapula, and the distal aspect of the left clavicle, right humerus and skull. Additionally, there is a focus of increased metabolic activity seen in the left posterior frontal lobe, revealing an intraparenchymal metastasis (see Figure 5). There are two hypermetabolic masses in the segment 4 of the liver and the one on the border of segment 4 a and segment 8 had a maximum SUV of 14. Multiple stones are identified within the gallbladder and common bile duct. There was a hypermetabolic lymph node seen at the porta hepatis, with maximum SUV of 9.2. There was a $1.6 \mathrm{~cm}$ hypermetabolic soft tissue nodule seen adjacent to the right colon with some surrounding enlarged mesenteric lymph nodes. 
Needle core biopsy of the gallbladder fossa mass showed poorly differentiated malignant neoplasm. The primary care location of the patient was Martinez VA and he was scheduled for Hematology/Oncology (Hem/Onc) consult there. The patient did not show up for the appointment and Hem/Onc recommended hospice care. The patient's condition deteriorated quickly and he expired on August 20, 2011.

Figure 1. This is a rotating 3D MIP image of the patient's FDG-PET/CT study showing numerous intensely hypermetabolic bony metastases.
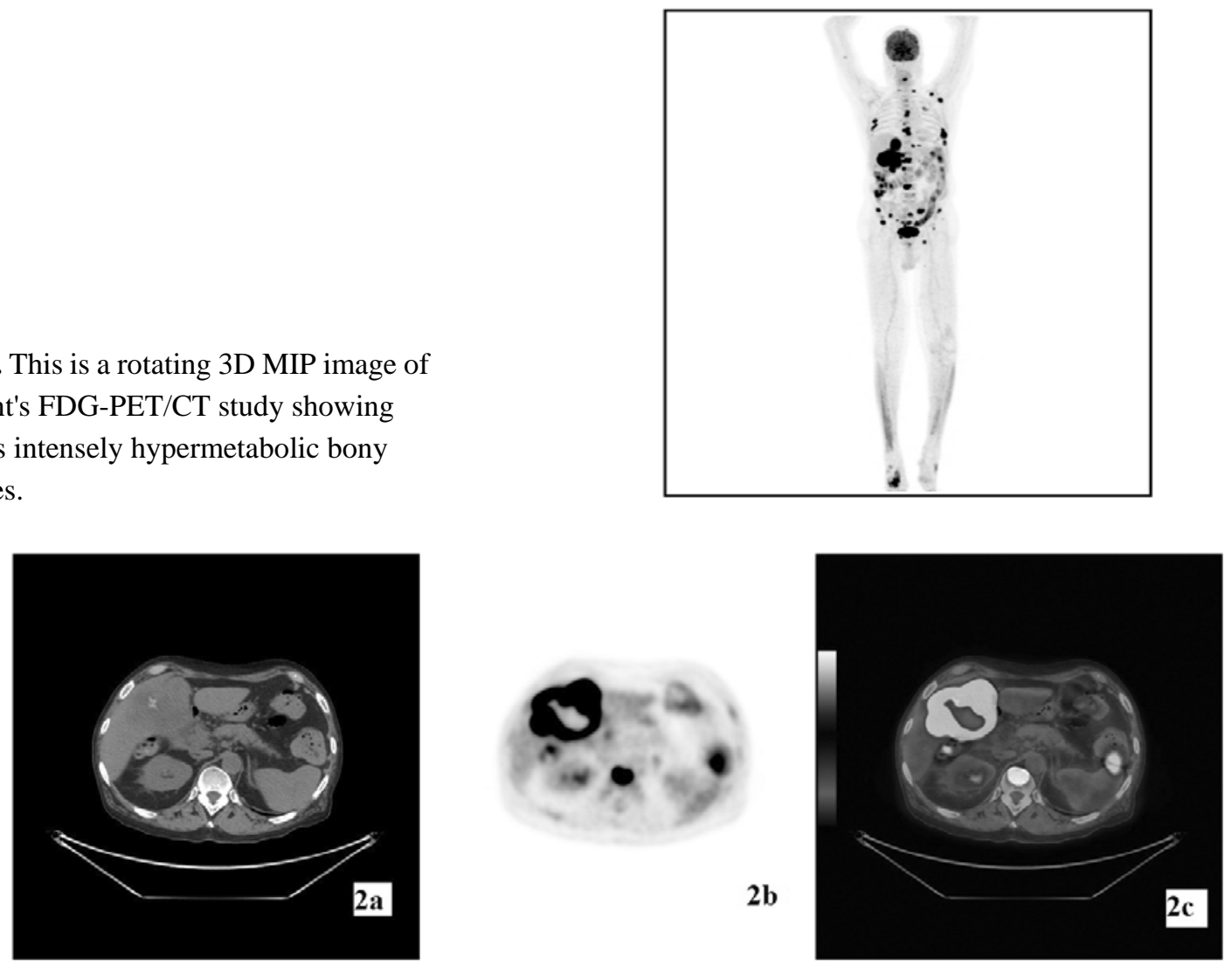

Figure 2. (a) CT transmission image for attenuation correction and anatomic localization, (b) FDG emission image and (c) hybrid FDG-PET/CT image of the L3 vertebra showing a intensely hypermetabolic bone metastases. Additionally, the gallbladder is enlarged and shows a intensely hypermetabolic and thickened wall with maximum SUV of 46, consistent with the patient's primary cancer.
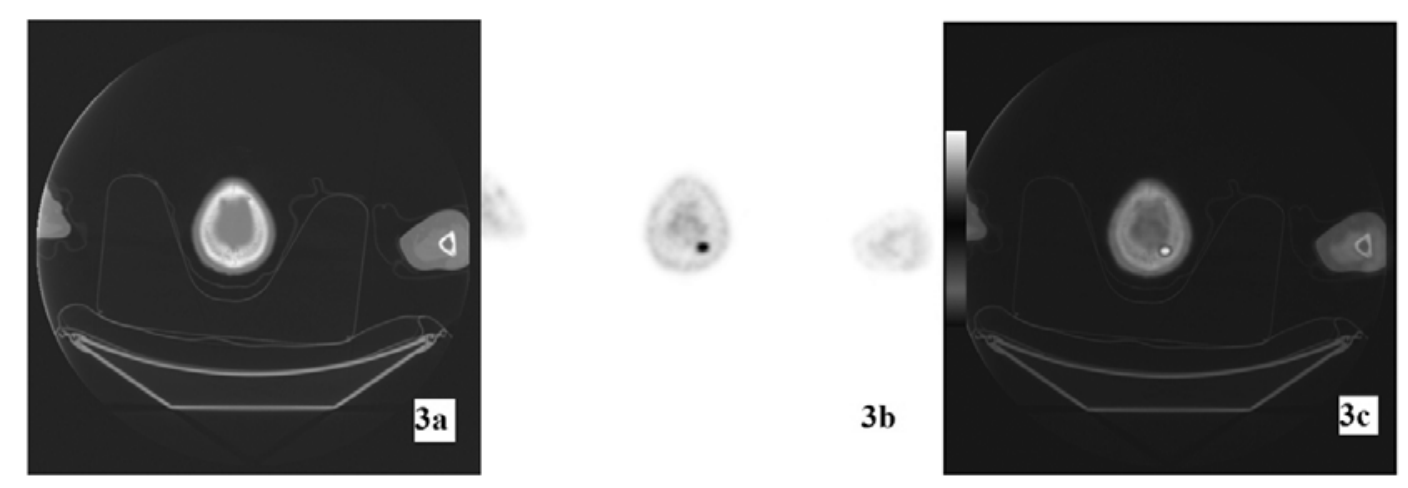

Figure 3. (a) This is the axial CT, for attenuation correction and antomic localization, showing normal thickness of the calvarium. (b) This is FDG emission image showing a focus of increased metabolic activity in the left posterior parietal calvarium near the vertex. (c) hybrid FDG-PET/CT image showing bony metastasis in the left posterior parietal calvarium. 

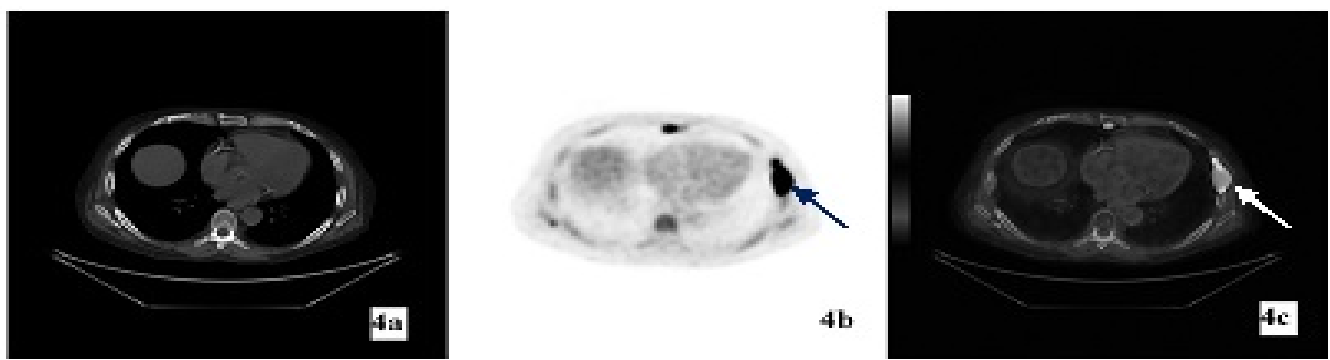

Figure 4. (a) This is the axial CT image, for attenuation correction and antomic localization, at the level of the heart. (b) FDG emission image showing a focus of increased metabolic activity (arrow) in the left rib. (c) hybrid FDG-PET/CT image showing a metabolically active lesion (arrow) in the left rib which represents a bony metastasis.

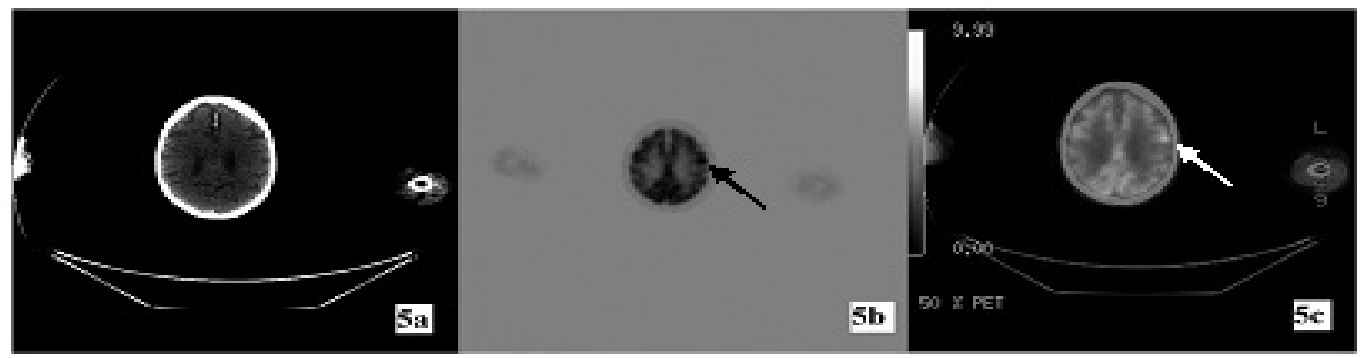

Figure 5. (a) This is the axial CT image, for attenuation correction and antomic localization, showing the brain parenchyma. (b) FDG emission image of the brain showing a focus of increased metabolic activity (arrow) in the left posterior frontal lobe. (c) hybrid FDG-PET/CT image showing an intraparenchymal metastasis (arrow) in the left frontal lobe.

\section{Discussion}

To our knowledge, this is the first report in the English language literature of gallbladder neoplasm metastasizing to both the bone (see Figure 1, 3, 4) and the brain parenchyma (see Figure 5). There are only 5 prior reports of gallbladder cancer metastasis to the bone in the English Language literature ${ }^{[3,11-14]}$. Likewise, gallbladder neoplasm metastasizing into the brain parenchyma is extremely rare. Our patient was an African American male and African Americans have the lowest rate of gallbladder cancer compared to other Americans ${ }^{[4]}$. The disease is 3 times more common in females than in males ${ }^{[2]}$. Risk factors for developing a gallbladder tumor include obesity, multiparity ( $>3$ ), gall stones, genetics, cholelithiasis, cholecystitis, and exposure to carcinogens ${ }^{[15]}$. Among them cholelithiasis is the most important risk factor, and up to $95 \%$ of patients with the disease have gallstones ${ }^{[15]}$. In this patient, there is presence of both cholelithiasis and choledocolithiasis. Larger stones $(>3 \mathrm{~cm})$ are associated with high risk of cancer ${ }^{[16]}$.

Ninety percent of the gallbladder tumors are adenocarcinomas ${ }^{[17]}$. Other types of tumor are squamous cell, adenosquamous, oat cell, and other anaplastic lesions, which occur rarely ${ }^{[4]}$. The gallbladder veins drain directly into segments 4 and 5 of the liver, where tumor invasion is common ${ }^{[4]}$. In this patient, liver metastasis is seen in segment 4 (see Figure 1). According to TNM staging, this tumor will qualify as T4N1M1 (stage IV) because it invaded the liver and mesenteric lymph nodes and distant metastases to the brain and skull are also present (see Figure 1 and 3). Consequently, they are all part of poor prognostic factors for the disease.

Additionally, CEA and CA 19-9 are nonspecific serum markers for gallbladder tumors ${ }^{[4]}$. Tumor markers are also used in monitoring the response to treatment and in following the progression of the cancers. In our patient, the CA 19-9 level is very high $(142.2 \mathrm{U} / \mathrm{ml})$, indicating an advanced disease. The average survival for patients with distant metastasis at the 
time of initial presentation is only 1 to 3 months ${ }^{[4]}$. Our case is in line with this prediction as the patient died about 5 weeks after the discovery of distant metastasis by FDG PET/CT. No oncologic treatment was planned for this patient. Quality of life has to balance with life expectancy and hospice care may be the best option in advanced cases such as this one.

The most common radiologic appearance of the gallbladder carcinoma is a mass replacing the gallbladder fossa ${ }^{[18]}$. However, CT cannot distinguish chronic cholecystitis with adjacent hepatic abscess from gallbladder neoplasm with liver metastasis ${ }^{[18]}$. In this patient, the prior CT scan could not confirm the presence of distant bone metastasis as there is normal bone density on CT. Yet, only on PET and hybrid PET/CT images, we can see metabolically active lesions in the brain and throughout the musculoskeletal system. Interestingly, the location of the brain metastasis in our case is in the left frontal lobe, which coincides with the case reports by Agrawal et al., Tayo et al., and Takano et al. ${ }^{[18-20]}$. Therefore, we can assume that left frontal lobe is a common location for gall bladder cancer metastasis in the brain. Gastrointestinal (GI) tumors are commonly detected by CT but in this case, the widespread bone metastasis is found by F18-FDG PET/CT which has higher sensitivity and higher positive predictive value than $\mathrm{CT}^{[21]}$. Several studies have highlighted the need to include FDG-PET/CT in the initial diagnosis of bladder cancer ${ }^{[13,14,22]}$. Since gallbladder cancer tends to present in late stages, we raise a question as to whether F18-FDG PET/CT scans be included in the routine workup protocol. Neoplasms in general, including gallbladder cancers, can present with nonspecific physical complaints such as in this case and it is important to rule out any metastases in the initial workup. Thus, this is a very rare case of gallbladder carcinoma metastasizing to the brain parenchyma and the skeletal system detected by NaF18-FDG PET/CT in an African American male.

\section{References}

[1] Grobmyer SR, Lieberman MD, Daly JM. Gallbladder cancer in the twentieth century: Single institution's experience. World J Surg. 2004; 28: 47. PMid:14639492 http://dx.doi.org/10.1007/s00268-003-7131-4

[2] Pandey M, Shukla VK. Diet and gallbladder cancer: A case-control study. Eur J Cancer Prev 2002; 11:365. PMid:12195163 http://dx.doi.org/10.1097/00008469-200208000-00008

[3] Prakash M, Aiyappan SK, Kumar A, et al. Solitary skeletal metastasis in carcinoma gallbladder: two case reports. Cancer Imaging. 2010; 10: 121-123. PMid:20529758 http://dx.doi.org/10.1102/1470-7330.2010.0014

[4] Wagholikar G, Behari A, Krishnani N, et al. Early gallbladder cancer. J Am Coll Surg. 2002; 194: 137. http://dx.doi.org/10.1016/S1072-7515(01)01136-X

[5] Kim JH, Kim TK, Eun HW. Preoperative evaluation of gallbladder carcinoma: Efficacy of combined use of MR imaging, MR cholangiography, and contrast-enhanced dual phase three dimensional MR angiography. J Magn Reson Imaging. 2002; 16 : 676. PMid:12451581 http://dx.doi.org/10.1002/jmri.10212

[6] Bartlett DL, Fong Y, Fortner JG, et al. Long-term results after resection for gallbladder cancer. Implications for staging and management. Ann Surg. 1996; 224: 639. PMid:8916879 http://dx.doi.org/10.1097/00000658-199611000-00008

[7] Wakai T, Shirai Y, Hatakeyama K. Radical second resection provides survival benefit for patients with T2 gallbladder carcinoma first discovered after laparoscopic cholecystectomy. World J Surg. 2002; 26: 867. PMid:11960212 http://dx.doi.org/10.1007/s00268-002-6274-z

[8] Chatni SS, Sainani RS, Mehta SA, et al. Infusion chemotherapy with cisplatinum and fluorouracil in the treatment of locally-advanced and metastatic gallbladder cancer. J Cancer Res Ther. 2008; 4: 151-155. PMid:19052386 http://dx.doi.org/10.4103/0973-1482.43341

[9] Iba T, Yagi Y, Kidokoro A, et al. Four cases of advanced adenocarcinoma showing marked response to EAP (etoposide, ADM, CDDP) treatment. Gan To Kagaku Ryoho.1993; 20: 2383-2385. PMid:8259856

[10] Tono T, Kim C, Takeda K, et al. Complete remission of liver metastasis from gall bladder carcinoma after stereotactic radiotherapy-a case report. Gan To Kagaku Ryoho. 2011; 38: 2103-2105. PMid:22202297

[11] Youssef F, Khan AW, Davidson BR. HPB (Oxford). 2003; 5(4): 258-60. Disseminated bony metastases following incidental gallbladder cancer detected after laparoscopic cholecystectomy. PMid:18332998 http://dx.doi.org/10.1080/13651820310001360

[12] Misra S, Chaturvedi A, Misra NC. Carcinoma gallbladder presenting with skeletal metastases. Indian J Gastroenterol. 1997 Apr; 16(2): 74. PMid:9114585

[13] Kumar A, Bhargava SK, Upreti L, et al. Disseminated osteoblastic skeletal metastasis from carcinoma gall bladder: a case report. Indian J Radiol Imaging. 2003; 13: 37-9. 
[14] Singh S, Bhojwani R, Singh S, et al. Skeletal metastasis in gallbladder cancer. HPB (Oxford). 2007; 9: 71-2. PMid:18333116 http://dx.doi.org/10.1080/13651820601110071

[15] Serra I, Calvo A, Baez S, et al. Risk factors for gallbladder cancer. An international collaborative case control study. Cancer. 1996; 78: 1515. http://dx.doi.org/10.1002/(SICI)1097-0142(19961001)78:7<1515::AID-CNCR21>3.0.CO;2-1

[16] Lowenfels AB, Walker AM, Althaus DP, et al. Gallstone growth, size, and risk of gallbladder cancer: An interracial study. Int J Epidemiol. 1998; 18: 50. http://dx.doi.org/10.1093/ije/18.1.50

[17] Levy AD, Murakata LA, Rohrmann CA. Gallbladder Carcinoma: Radiologic-Pathologic Correlation. AFIP Archives. 2001; 21: 295-312.

[18] Agrawal A, Agrawal CS, Kumar A, et al. Gall bladder carcinoma: stroke as first manifestation. Indian Journal of Gastroenterology. 2006; 25: 316. PMid:17264439

[19] Tayo JR, Al-Abdulkarim H, Al-Rayess M. Brain metastasis as an initial manifestation of a gallbladder carcinoma. Neurosciences. 2005; 10: 235-237. PMid:22473267

[20] Takano S, Yoshii Y, Owada T, Shirai S, Nose T. Central nervous system metastasis from gallbladder carcinoma--case report.Neurol Med Chir (Tokyo). 1991 Dec; 31(12): 782-6. Review. http://dx.doi.org/10.2176/nmc.31.782

[21] Minamimoto R, Senda M, Terauchi T, et al. Analysis of various malignant neoplasms detected by FDG-PET cancer screening program: based on a Japanese Nationwide Survey. Ann Nucl Med. 2011; 25: 45-54. PMid:20953985 http://dx.doi.org/10.1007/s12149-010-0428-0

[22] Butte JM, Redondo F, Waugh E, Meneses M, Pruzzo R, Parada H, Amaral H, De La Fuente HA. The role of PET-CT in patients with incidental gallbladder cancer. HPB (Oxford). 2009 Nov; 11(7): 585-91. PMid:20495711

http://dx.doi.org/10.1111/j.1477-2574.2009.00104.x 